\title{
Efficacy and safety of parecoxib for the treatment of pain following total knee arthroplasty in Korean patients
}

M.N. Essex, ${ }^{1}$ H.-Y. Choi, ${ }^{2}$ P. Bhadra Brown, ${ }^{1}$ R. Cheung ${ }^{1}$

${ }^{1}$ Pfizer Inc, New York, NY, USA; ${ }^{2}$ Pfizer Pharmaceutical Korea Ltd, Seoul, South Korea

\section{INTRODUCTION}

Korea had a growth rate of $407 \%$ in total knee arthroplasty (TKA) procedures from 2001 to 2010.1 More than half of patients undergoing TKA experience severe postoperative pain. ${ }^{2}$ Several factors, including ethnicity, can influence patient perception of pain..$^{3,4}$

The current clinical recommendation for postoperative pain is a multimodal analgesic approach intended to efficiently manage pain and to reduce opioid use and opioid-related adverse events (AEs). ${ }^{2,5}$

Parecoxib is an injectable cyclooxygenase-2 inhibitor with proven efficacy for postoperative pain, including gynaecological ${ }^{6}$ and oral surgery, ${ }^{7}$ and total hip or knee replacement. ${ }^{8-11}$

The effect of ethnicity on the analgesic efficacy of parecoxib has not been extensively studied.

The current study assessed the analgesic efficacy and safety of intravenous (IV) parecoxib sodium $40 \mathrm{mg}$ vs. placebo for post-TKA pain in Korean patients.

\section{METHODS}

This parallel-group, double-blind, randomised study was conducted at 4 centres in Korea and in accordance with International Conference on Harmonisation Good Clinical Practice guidelines, the Declaration of Helsinki, and applicable local regulatory requirements and laws.

Each patient provided written consent to participate in the study prior to enrolment.

Men and women aged $\geq 18$ years who had undergone unilateral TKA performed under general, regional, or spinal anaesthesia were enrolled in the study.

Patients were given postoperative analgesia provided by morphine or meperidine, administered by patientcontrolled analgesia (PCA).

Those patients with moderate or severe pain within $6 \mathrm{~h}$ after the end of PCA were randomised to receive a single IV dose of parecoxib sodium $40 \mathrm{mg}$ or placebo the day after surgery.

The primary efficacy variables were:

Time-specific pain intensity difference (PID) on a 4-point self-rated scale ( $0=$ none to $3=$ severe).

PID was derived by subtracting the pain intensity scores for each time point post-dose from the baseline score.

Time-specific pain relief $(P R)$ on a 5 -point self-rated scale ( $0=$ none to $4=$ complete).

Time-specific sum of PID and PR (PRID).

Time to rescue medication, which was provided at the investigator's discretion.

Efficacy measures were assessed at baseline (time 0 ) and 0.25, 0.5, 0.75, 1, 1.5, 2, 3, 4, 5, 6, 7, 8, 10, 12, 16 , and $24 \mathrm{~h}$ after dosing, and just prior to taking rescue medication if this was used.

- PID, PR, and PRID were analysed using analysis of covariance (ANCOVA) with treatment and centre as factors and baseline pain intensity as a covariate.

The median time to rescue medication, with corresponding 95\% confidence intervals, was determined using the Kaplan-Meier product limit estimator, with distributions analysed using a log-rank test.

Safety was assessed by the incidence and nature of AEs and analysed using Fisher's exact test.

\section{RESULTS}

116 patients were randomised; 58 received parecoxib and 58 received placebo.

There were no significant differences between treatment groups in patient demographic and baseline

characteristics, including duration of surgery, duration of anaesthesia, and baseline pain severity

(data not shown).

\section{Efficacy}

Mean PID was significantly greater for parecoxib vs. placebo starting at 1-h postdose and continuing throughout the 24-h treatment period (all $p<0.05$ ) (Figure 1).

Figure 1. Change in mean pain intensity difference, by treatment group

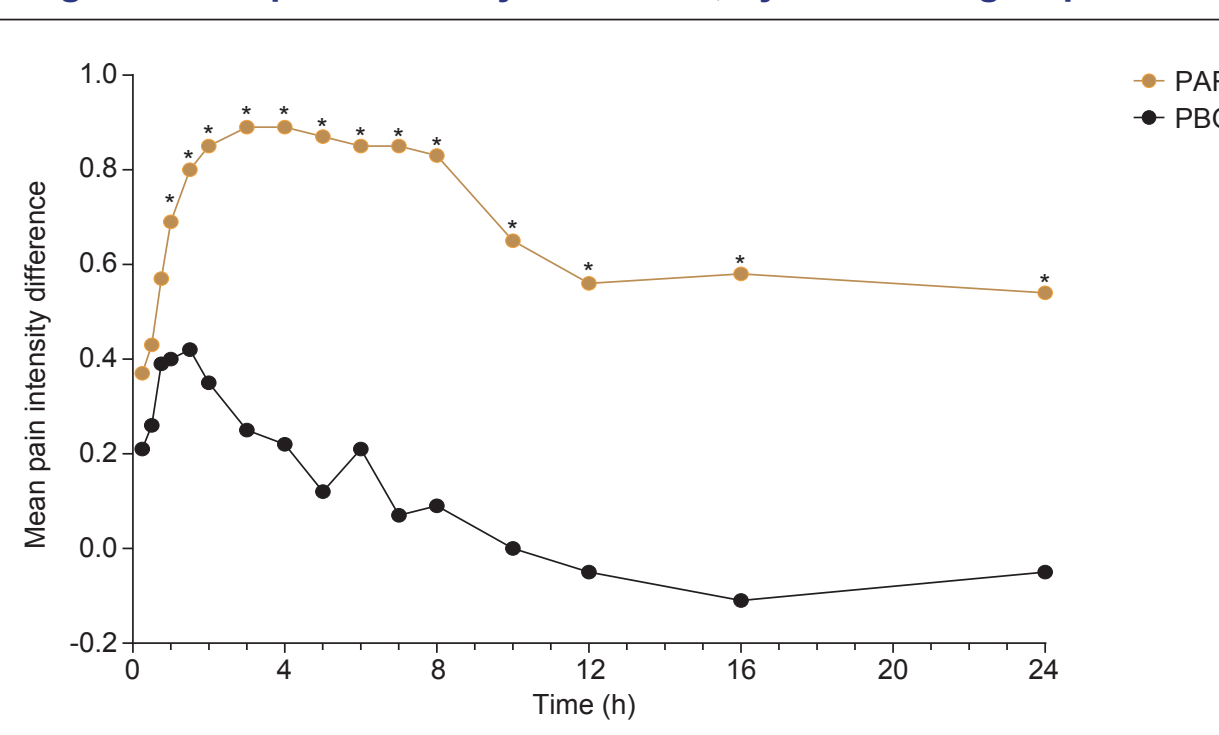

* $p<0.05$ for parecoxib vs. placebo for each time point

Mean PR was significantly greater for parecoxib vs. placebo, beginning at 1.5 -h postdose and continuing throughout the 24-h treatment period (all $p \leq 0.001$ ) (Figure 2).

Mean PRID was significantly greater for parecoxib vs. placebo starting at 1 -h postdose and continuing throughout the 24-h treatment period (all $p<0.05$ ) (Figure 3).

Over the 24-h treatment period, 28 of 54 (52\%) patients in the parecoxib group required rescue medication vs. 51 of $57(89 \%)$ patients in the placebo group $(p<0.001)$.

The median time to rescue medication was significantly longer for parecoxib $(21.5 \mathrm{~h})$ vs. placebo $(4.1 \mathrm{~h} ; p<0.001)$ (Figure 4).
Figure 2. Change in mean pain relief, by treatment group

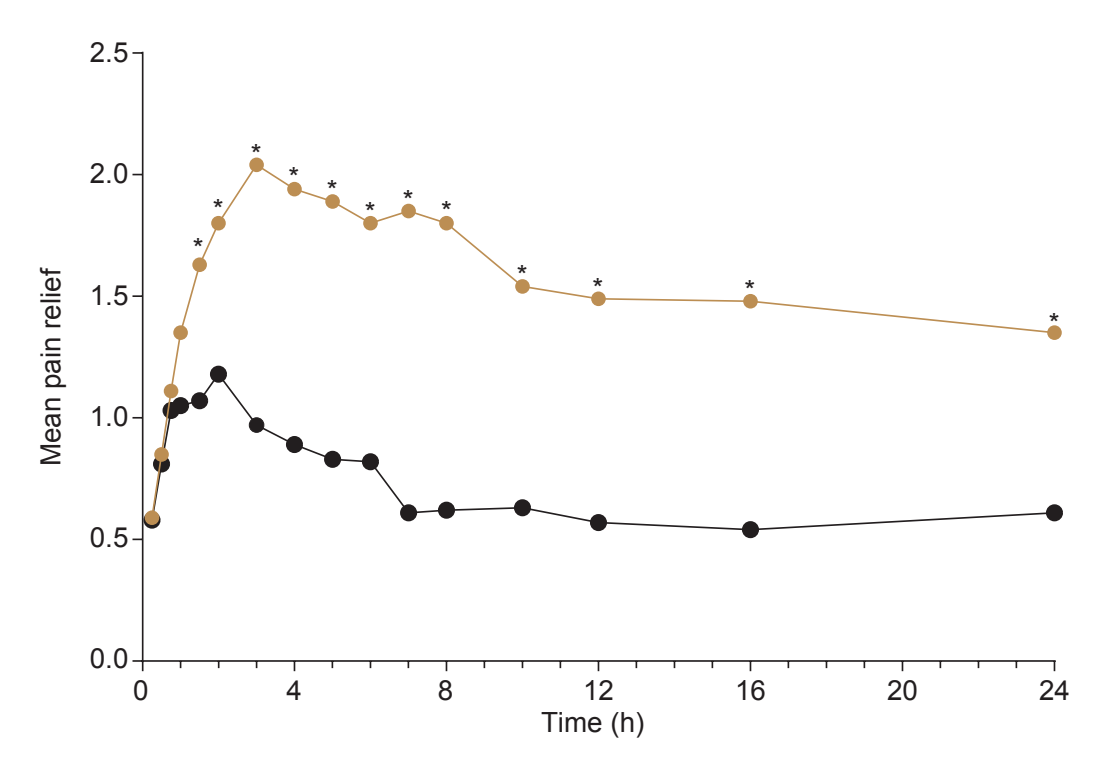

p $\leq 0.001$ for parecoxib vs. placebo for each time point. A higher score indicates greater pain relief vs. baseline (time 0 ).

\section{$\mathrm{PAR}=$ parecoxib; $\mathrm{PBO}=$ placeb}

Figure 3. Change in mean time-specific sum of pain intensity difference and pain relief

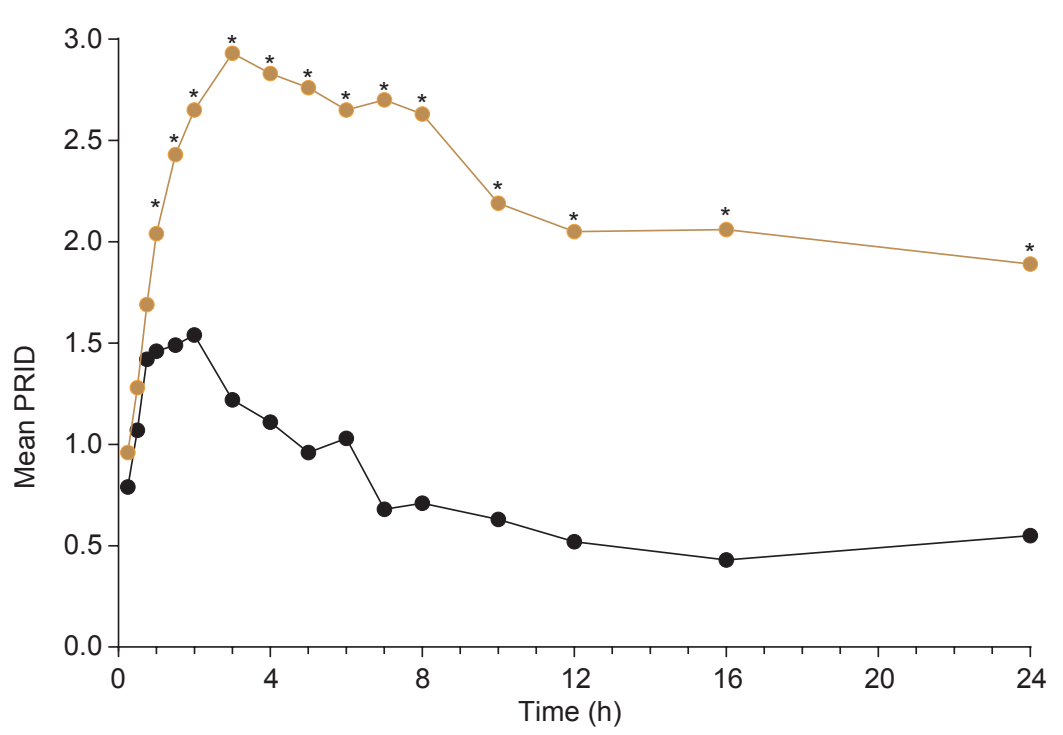

* $p<0.05$ for parecoxib vs. placebo for each time point.

A higher score indicates greater pain relief vs. baseline (time 0 ).

Figure 4. Median time to rescue medication

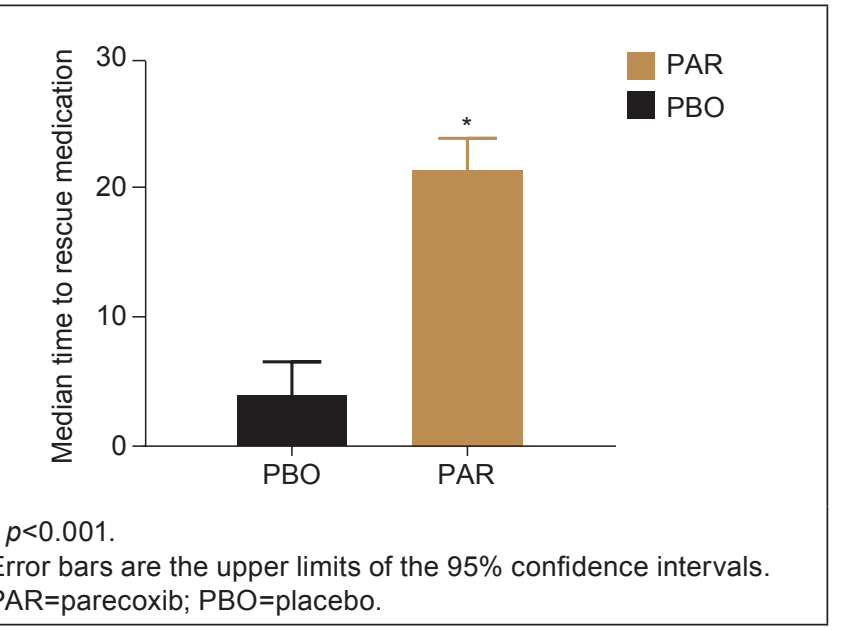

Safety

$22(37.9 \%)$ patients in the placebo treatment group and $12(20.7 \%)$ patients in the parecoxib AEs (Table 1).

No serious AEs or deaths were reported.

AEs were generally mild to moderate in severity. 1 incidence of pruritus in the parecoxib group was judged as severe by the investigator, but resolved within $2 \mathrm{~h}$ and was not considered to be treatment-related.

Of the treatment-emergent AEs, 2 incidences of pruritus in the parecoxib group were deemed treatment-related by the investigator.

Table 1. Treatment-emergent adverse events occurring in $\geq 3 \%$ of patients in either treatment group

\begin{tabular}{lcc}
\hline Adverse Event, $\mathbf{n}(\%)$ & $\begin{array}{c}\text { Placebo } \\
\mathbf{n}=58\end{array}$ & $\begin{array}{c}\text { Parecoxib } \\
\mathbf{n}=58\end{array}$ \\
\hline All & $22(37.9)$ & $12(20.7)^{\star}$ \\
Pruritus & $0(0.0)$ & $4(6.9)$ \\
Headache & $4(6.9)$ & $2(3.4)$ \\
Constipation & $0(0.0)$ & $2(3.4)$ \\
Dyspepsia & $2(3.4)$ & $2(3.4)$ \\
Hypertension & $4(6.9)$ & $1(1.7)$ \\
Nausea & $5(8.6)$ & $1(1.7)$ \\
Vomiting & $2(3.4)$ & $1(1.7)$ \\
Fever & $7(12.1)$ & $0(0.0)^{*}$ \\
Abdominal pain & $2(3.4)$ & $0(0.0)$ \\
\hline${ }^{*}$ p<0 & &
\end{tabular}

${ }^{*} p<0.05$.

\section{CONCLUSIONS}

In Korean patients with moderate or severe pain after TKA, a single IV dose of parecoxib sodium $40 \mathrm{mg}$ provided significantly greater pain relief for a significantly longer time vs. placebo.

Few treatment-related AEs were reported in either parecoxib- or placebo-treated patients.

These results are comparable to those from other studies demonstrating the analgesic efficacy and safety of parecoxib for post-TKA pain in non-Korean patient populations. ${ }^{9-11}$

Korean ethnicity appears to have no impact on the efficacy or safety profile of parecoxib when used for treating post-TKA pain. 